\title{
Turning with the others: novel transitions in an SPP model with coupling of accelerations
}

\author{
Péter Szabó ${ }^{\dagger \dagger}$, Máté Nagy ${ }^{\dagger}$, and Tamás Vicsek ${ }^{\dagger \ddagger}$
}

\begin{abstract}
†Department of Biological Physics, Eötvös University, Pázmány Péter sétány 1A, H-1117 Budapest, Hungary ${ }^{\ddagger}$ Statistical and Biological Physics Research Group of the Hungarian Academy of Sciences, Pázmány Péter sétány 1A, H-1117 Budapest, Hungary ${ }^{\dagger}$ To whom correspondence should be addressed. E-mail:pszabo@angel.elte.hu.
\end{abstract}

\begin{abstract}
We consider a three dimensional, generalized version of the original SPP model for collective motion. By extending the factors influencing the ordering, we investigate the case when the movement of the self-propelled particles (SPP-s) depends on both the velocity and the acceleration of the neighboring particles, instead of being determined solely by the former one. By changing the value of a weight parameter $s$ determining the relative influence of the velocity and the acceleration terms, the system undergoes a kinetic phase transition as a function of a behavioral pattern. Below a critical value of $s$ the system exhibits disordered motion, while above it the dynamics resembles that of the SPP model. We argue that in nature evolutionary processes can drive the strategy variable $s$ towards the critical point, where information exchange between the units of a system is maximal.
\end{abstract}

\section{Inroduction}

Collective motion of organisms (e.g. fish schools, bird flocks, bacterial colonies) exhibits a large variety of emergent phenomena [1, 2, 3, 4, 5, 6, 7, 8]. Synchronized motion, symmetrical group formations (e.g., V shaped) or swirling patterns emerge in spite of the apparently simple behavioral rules of the individual flock members [9, 10]. The self propelled particles (SPP) model was proposed by Vicsek et al. [11] to describe the onset of ordered motion within a group of self-propelled particles in the presence of perturbations. Taking into the effects of fluctuations inevitably present in biological systems was an essential generalization of the prior deterministic flocking models such as that of Reynolds [12]. The original model considers point-like particles moving at constant velocity on a two dimensional surface with periodic boundary conditions. The only rule is that, at each time step, every particle approximates, with some uncertainty, the average direction of motion of the particles within its neighborhood of radius $R$. This model exhibits spontaneous self-organization; by decreasing the noise parameter, the system undergoes a kinetic phase transition from a disordered state to an ordered one where all the particles move approximately in the same direction. Due its simplicity and analogy with biological systems comprised of many, locally interacting particles, the SPP model soon became a reference model for the flocking behavior of organisms [13, 14, 15, 16, 17, 18, 19, 20, 21, 22, 23].

The individual based behavioral rules, determining collective motion, are of particular interest. Important elements of behavioral rules are the nature of the perceived information and the affected behavioral traits. [24, 25, 26]. A frequent assumption in models is that the information, perceived by the particles, is restricted to the velocity of their neighbors. The interaction range is usually defined by metric distances, but Ballerini et al. [24] recently showed that topological distance is the one determining the flocking of starlings. The assumption of reflecting on the momentary velocity only may not be enough for adequately describing a number of biologically relevant situations. We expect that the behavior of 
the SPP model is significantly extended if we also incorporate a term corresponding to memory on short time scales. This can be achieved by introducing an acceleration term into the equations (rules). This is equivalent to separating time scales by assuming that the particles differentiate between two kind of information, the first being their actual velocity, the second one corresponding to recent change in their direction of motion. For example, in the case of birds, reacting to acceleration may mean that birds can give signals to their neigbors about their intended changing of their flight direction by quickly modifying their velocity.

\section{Model}

In the three-dimensional, scalar noise (SNM) version [27] of the original SPP model [11] the particles are assumed to move with a constant velocity $\nu$, and their positions are updated simultaneously according to

$$
\mathbf{x}_{\mathbf{i}}(t+\Delta t)=\mathbf{x}_{\mathbf{i}}(t)+\mathbf{v}_{\mathbf{i}}(t) \Delta t,
$$

where $\mathbf{x}_{i}$ and $\mathbf{v}_{i}$ are position and velocity of particle $i$, respectively. The time increment is set to be $\Delta t=1$. Each particle is assumed to move, with some uncertainty, in the average direction of all the particles within a fixed neighborhood of radius $R=1$. Hence, the new velocity is given by

$$
\mathbf{v}_{\mathbf{i}}(t+\Delta t)=\nu \cdot \mathcal{M}(\mathbf{e}, \xi) \cdot \mathbf{N}\left(\langle\mathbf{v}(t)\rangle_{i, R}\right),
$$

where $\nu$ is the absolute value of velocity, $\mathcal{M}(\mathbf{e}, \xi)$ is a rotational tensor representing a random perturbation, $\langle\mathbf{v}(t)\rangle_{i, R}$ denotes the average velocity of all particles around particle $i$ within radius $R$ including particle $i$ itself, and $\mathbf{N}(\mathbf{u})=\mathbf{u} /|\mathbf{u}| . \mathcal{M}(\mathbf{e}, \xi)$ performs a rotation of angle $\xi$ around a vector $\mathbf{e} ; \xi$ is a uniform random value in the interval $[-\eta \pi, \eta \pi]$, whereas $\mathbf{e}$ is a random unit vector chosen uniformly from the set of vectors perpendicular to $\mathbf{N}\left(\langle\mathbf{v}(t)\rangle_{i, R}\right)$. The order in which $(1)$ and 22 are calculated has some quantitative effects on the results (see later).

Here we introduce the acceleration coupled self-propelled particles model (AC-SPP) being a modified version of SNM, in which the velocity vector $\mathbf{v}_{\mathbf{i}}(t+\Delta t)$ is a function of both the velocity $\mathbf{v}(t)$ and the acceleration $\mathbf{a}(t)=(\mathbf{v}(t)-\mathbf{v}(t-\Delta t)) / \Delta t$ of the neighboring particles. Then equation 2 becomes

$$
\mathbf{v}_{\mathbf{i}}(t+\Delta t)=\nu \cdot \mathcal{M}(\mathbf{e}, \xi) \cdot \mathbf{N}\left(s \cdot\langle\mathbf{v}(t)\rangle_{i, R}+(1-s) \cdot\langle\mathbf{a}(t) \Delta t\rangle_{i, R}\right),
$$

where $s \in(0,1]$ is a so-called strategy parameter, expressing the relative influence of the acceleration and velocity tags on the velocity vector of the focal particle. Initially, we have $N=\rho L^{3}$ randomly distributed particles, where $L$ and $\rho$ stand for box size and particle number density, respectively. The bounding box has periodic boundary conditions. The velocity parameter used in the simulations is $\nu=0.1$, corresponding to the low velocity regime [21].

Taking into account the acceleration in a separate term has various possible motivations. As for the contribution of the $i$-th particle, it corresponds to a memory effect: a given particle, if it has no neighbors, has a tendency to keep on turning as it did in the previous time step. Perhaps more importantly, the average turning rate of the neighbors has now a separated effect on the turning rate of the $i$-th particle. In the $s$ close to 1 limit the AC-SPP model is very much like the original SPP, while for $s \ll 1$ the acceleration term dominates and instantaneous turning of the neighbors has a strong effect on the trajectory of the $i$-th particle. In this way not only the state (velocity), but a kind of behavioral pattern (turning) can be taken into account. We associate, for example, with such a behavioral pattern a short turning period of a bird flying in a flock and giving sign for its neighbors of its intended changing of the direction of flight.

We characterize the collective motion of particles by the average velocity of all particles $\varphi$, defined as

$$
\varphi=\frac{1}{N}\left|\sum_{i=1}^{N} \mathbf{v}_{\mathbf{i}}\right|,
$$


with $N$ denoting the number of particles in the system. This order parameter can take any value in the range $[0,1]$, and expresses the tendency of particles to move in the same direction. If the particles move randomly $\varphi=0$ whereas if every particle moves in the same direction $\varphi=1 .\langle\varphi\rangle$ was obtained by averaging over 12 individuals runs, each recorded after a relaxation time to stationarity $T_{\text {relax }}$ and with time averaging over an additional $T_{\text {avr }}$ time steps.

\section{Results}

At first we investigated the dynamics of the system at different values of the strategy variable $s$ at fixed density and noise values: $\rho=0.16, \eta=1 / 9$. The density was choosen to be high enough to get flocking, but allowing moderate CPU times per run. The noise value was a typical value resulting in an ordered state for the original SPP model $(s=1)$. We found that by increasing $s$ the system undergoes a phase transition; below a critical value, $s_{c}$, the ordering, expressed by $\varphi$, is negligible, while above $s_{c}$ the level of order increases rapidly as a function of $s$ (Figure 1). This is a novel type of phase transition since it corresponds to a phase change due to a change in the relative strength of a behavioral pattern.

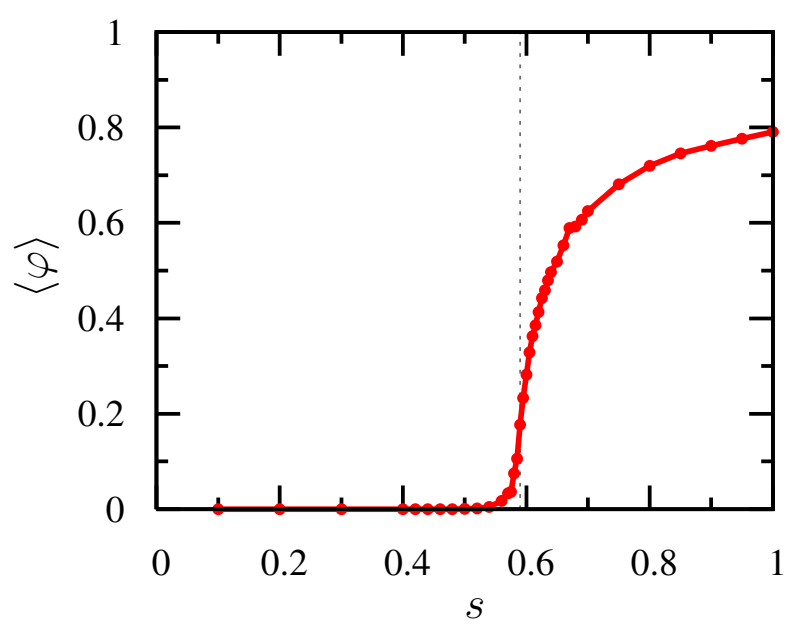

Figure 1: Average velocity $\varphi$ as a function of the strategy variable $s$. The data points were obtained by averaging the results of 12 simulations, using the parameters $L=100, \rho=0.16, \nu=1 / 9$, with a relaxation time of $T_{\text {relax }}=10000$ time steps and averaging over an additional $T_{\text {avr }}=10000$ time steps.

In order to determine the nature of the phase transition we also calculated the probability density function (PDF) of $\varphi$ and the Binder cumulant $G$ at different strategy values. The Binder cumulant, defined as $G=1-\left\langle\varphi^{4}\right\rangle / 3\left\langle\varphi^{2}\right\rangle^{2}$, measures the fluctuations of the order parameter and can be used to distinguish between first- and second order phase transitions [31]. In case of a first order phase transition $G$ exhibits a characteristic minimum, whereas in case of a second order transition this sharp minimum is absent. In our case, the PDF was unimodal, and $G$ did not have a sharp minimum around $s_{c}$, both indicating a second order phase transition (Figures 2 and 33. $G$ was monotonously increasing, as it was observed already in a three dimensional SNM with continuous phase transition [27].

Consequently, near the critical point, the order parameter obeys the scaling relation

$$
\varphi \sim\left[s-s_{c}(\rho, \eta)\right]^{\beta},
$$

where $\beta$ is the critical exponent. The critical values $s_{c}$ and $\beta$ were determined by plotting $\log \langle\varphi\rangle$ as a function of $\log \left[\left(s-s_{c}\right) / s_{c}\right]$ (Figure 4). $s_{c}$ was obtained by finding the value where the plot was the 


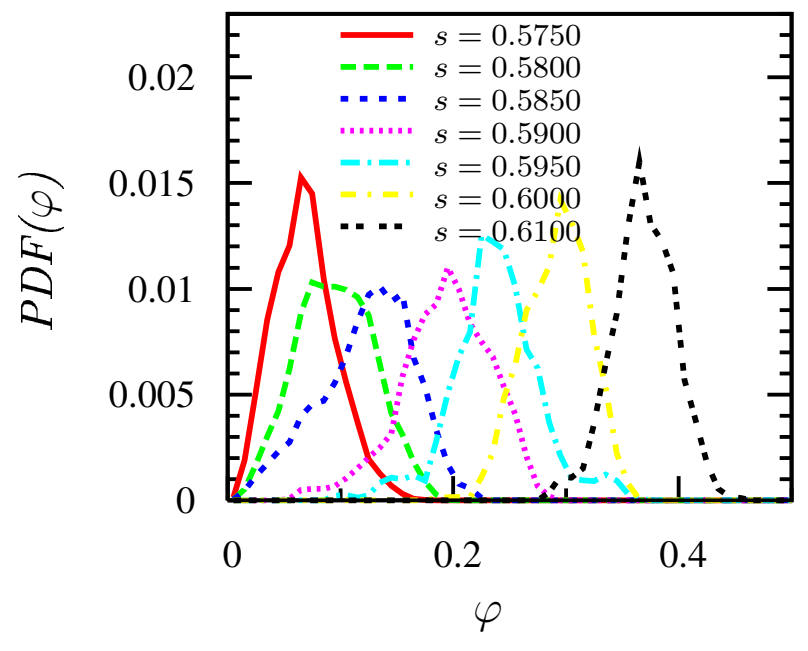

Figure 2: Probability density function (PDF) of the order parameter $\varphi$. Parameters as in the previous plot. The one-humped distributions in a range of $s$ values close to the critical one $\left(s_{c} \simeq 0.59\right)$ suggest a second order phase transition.

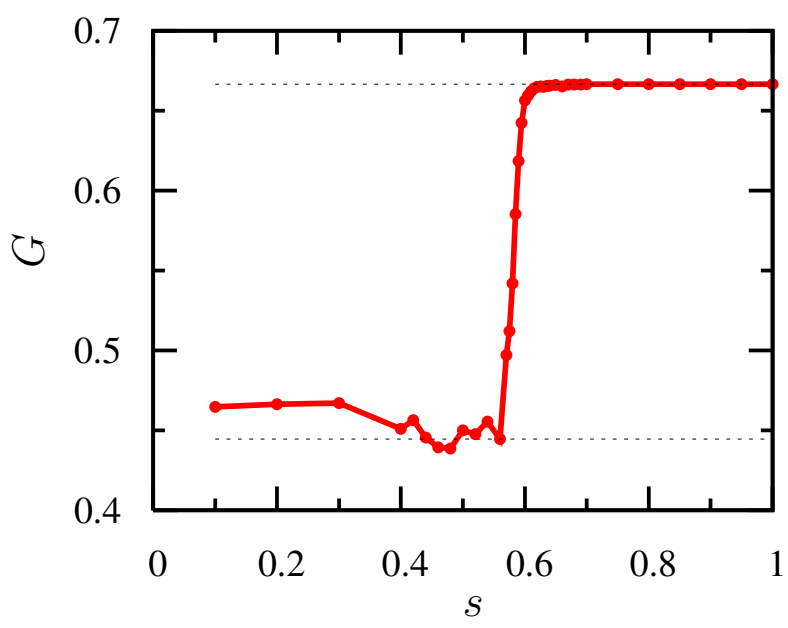

Figure 3: The Binder cumulant $G$ as a function of the strategy variable $s$. The dotted lines at $2 / 3$ and $4 / 9$ indicate the theoretical value in the case of the ordered and totally disordered states, respectively. Parameters as in the previous plots. The absence of a sharp minimum indicates a second order phase transition. 
straightest in the relevant region, whereas the critical exponent is equal to the slope of the fitted line. We obtained $s_{c}=0.590 \pm 0.002$ and $\beta=0.35 \pm 0.05$. It should be noted, that the value $\beta=0.35$ is far from 0.5 (corresponding to mean-field or bifurcation) indicating the true non-trivial nature of the transition we describe.

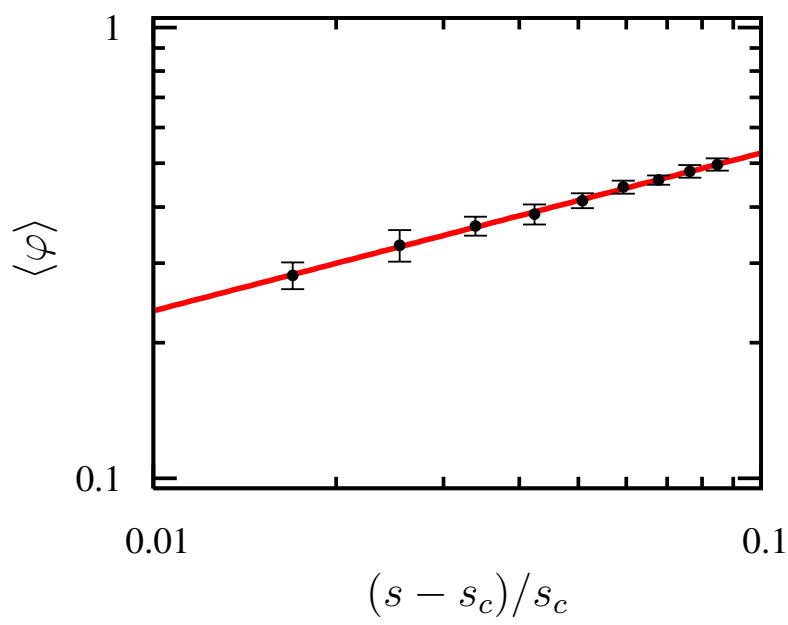

Figure 4: Average velocity values as a function of the distance from the critical point. The fitted line, with $s_{c}=0.590 \pm 0.002$, had a slope of $\beta=0.34 \pm 0.05$. Parameters as in the previous plots.

The critical value of the transition depends on both the density and the noise parameters. Figure 5 shows that $s_{c}$ is decreasing with both increasing density and noise values.

The order variable $\varphi$, by itself, provides a poor description of the two states, hence we also calculated other statistics. The sinuosity of the particle trajectories was expressed by their average curvature, defined as $\lambda=\frac{1}{N} \sum_{N} \frac{\left|v_{i} \times a_{i}\right|}{\left|v_{i}\right|^{3}}$. Two other statistics, $\psi$ and $\mu$, measure the information exchange between the particles. $\psi$ is defined as the average number of different particles encountered, i.e., being within a distance $R$, by a focal particle during a given time interval. Although this quantity depends on the chosen time interval, its monotonicity as a function of $s$ is independent of it. $\mu$ was used to evaluate the speed of information propagation, as follows. Initially one percent of the particles held the information. The information was transmitted between particles via encounters between information holders and other particles. This way sooner or later every particle became information holder. $\mu$ was defined as the time needed for at least 90 percent of the particles becoming information holders.

All the three statistics $\langle\lambda\rangle,\langle\psi\rangle$ and $\langle\mu\rangle$ were obtained by averaging over 12 individual runs, each with a relaxation time of $T_{\text {relax }}=10000$ and averaging time of $T_{\text {avr }}=10000 .\langle\psi\rangle$ is an average value for 100 randomly chosen particles.

The curvature of the trajectories decreases with the strategy variable (Figure 6). Large curvature at low $s$ values indicates in this case, that particles move in small circles (Figure 7). It is because the large influence of the acceleration term results in continuous turning. This turning is synchronized among neighbors, i.e., their acceleration and velocity vectors become the same, resulting in a particle cloud consisting of separated groups of particles, each containing circling particles. By increasing $s$ the radius of these circles increases, until the circling groups overlap and start to interact with each other. At a critical point the circles overlap so much, that neither their position, nor their composition remains the same; in other words the circling groups lose their identity and the particles start to move sinuously. At large $s$ values the movement becomes ordered; all particles tend to move in the same direction, similar to the ordered phase of the SNM model at small velocities.

The dynamics of the system is well reflected in the information propagation (Figure 8). Both $\psi$ and $\mu$ 


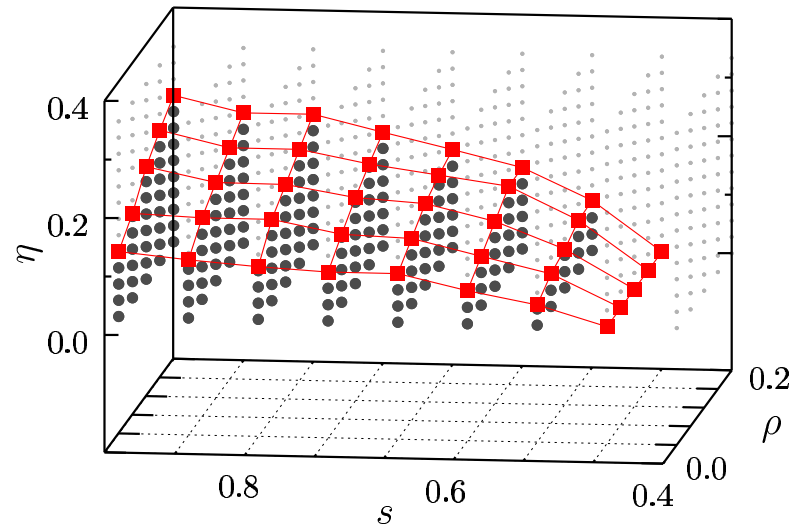

Figure 5: Border surface between the ordered and disordered phases in the $s, \rho, \eta$ parameter space. The ordered $(\langle\varphi\rangle \geq 0.01)$ and disordered $(\langle\varphi\rangle<0.01)$ states are indicated by heavy and light dots, respectively. Squares show the critical noise values $\eta_{c}(s, \rho)$ obtained from the plot of $\log \langle\varphi\rangle$ as a function of $\log \left[\left(\eta_{c}-\eta\right) / \eta_{c}\right]$ for each $s$ and $\rho$ pair. $L=100$.

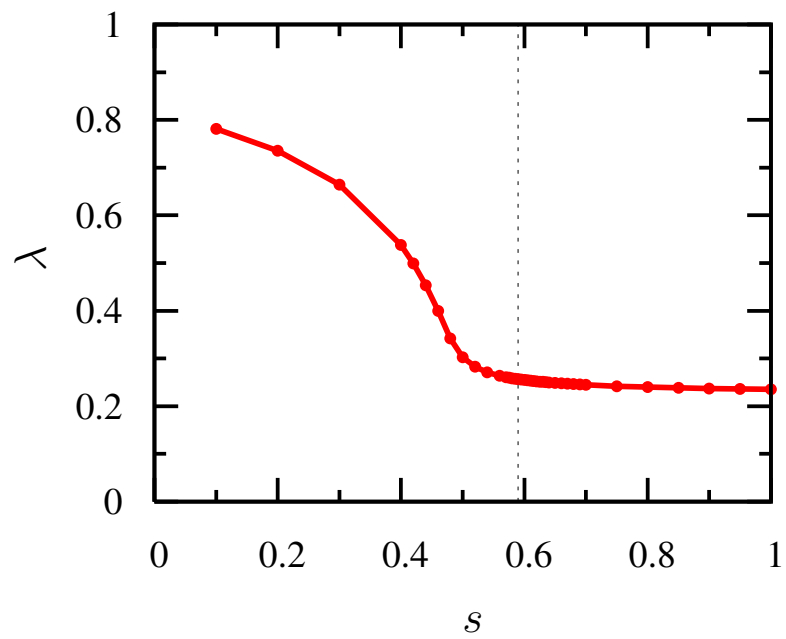

Figure 6: The curvature of trajectories $\lambda$ as a function of the strategy variable $s$. The dotted line at $s_{c}=0.590$ indicates the position of the critical point for ordering. Parameters as in the previous plots. 

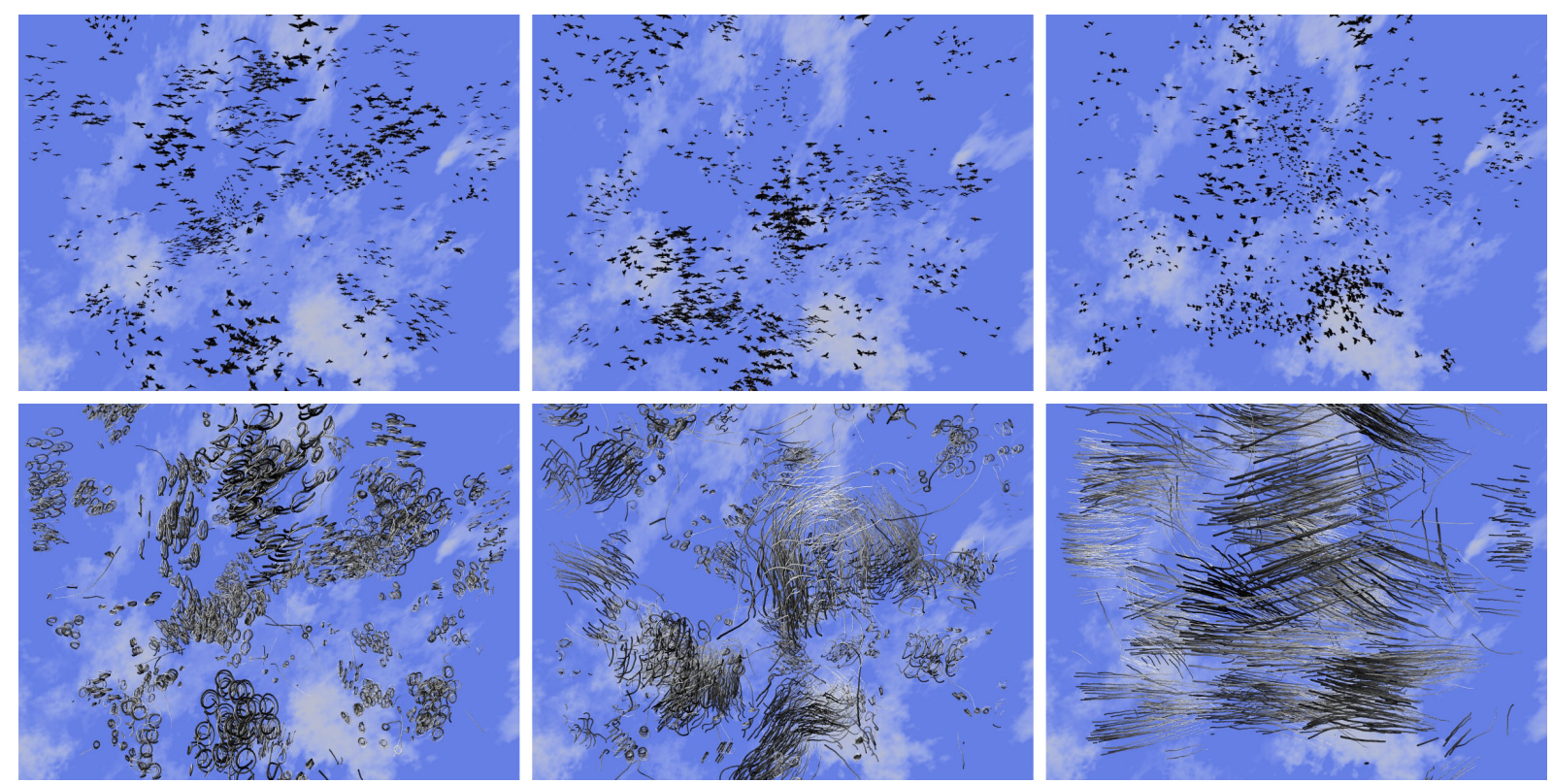

Figure 7: Positional data and spatial trajectories of particles at different strategy values. Subfigures show the typical behavior (a, d) below, (b, e) near and (c, f) above the critical point, respectively. (a-c) Positional data of the particles shown as birds. (d-f) Each curve shows the trajectory of a particle over 60 time steps after reaching steady state $(t=20000)$. Different shades of gray indicate the time past, with darker tones denoting more recent positions. (a, d) Below the critical point, at $s=0.5$ the particles move in circles. (b, e) Near the critical point, at $s=0.53$, the particles move sinuously. (c, f) Above the critical point, at $s=0.9$, the particles move in the same direction. $L=20, \eta=1 / 6$, other parameters as in the previous plots.

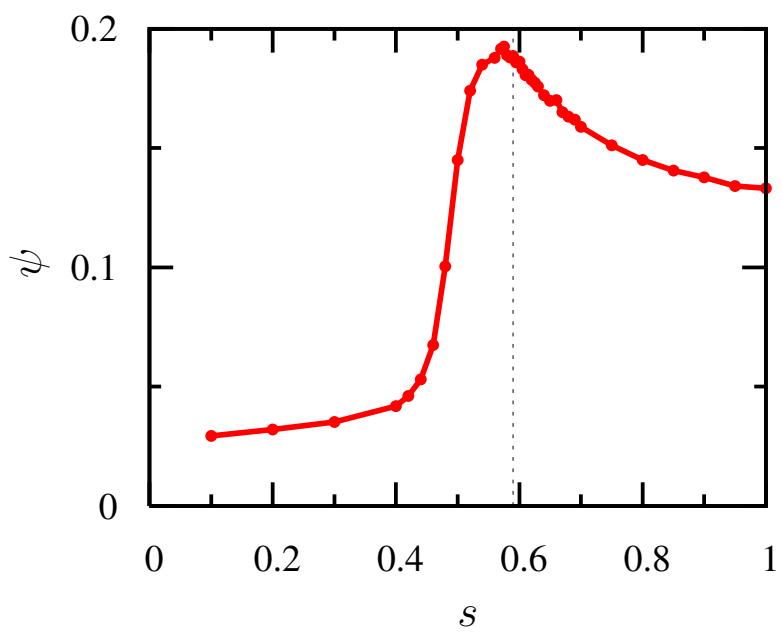

Figure 8: Information exchange between particles $(\psi)$ as a function of the strategy variable $s$. The curve for $\mu$ was very similar (not shown). Parameters as in Figure 1 . 
have low values at small $s$ and have a maximum value around $s_{c}$ (see also Ref. [32]). This result held for all density and noise parameter values investigated. The curves of $\psi$ and $\mu$ were very similar, indicating that both are proper measures of information propagation.

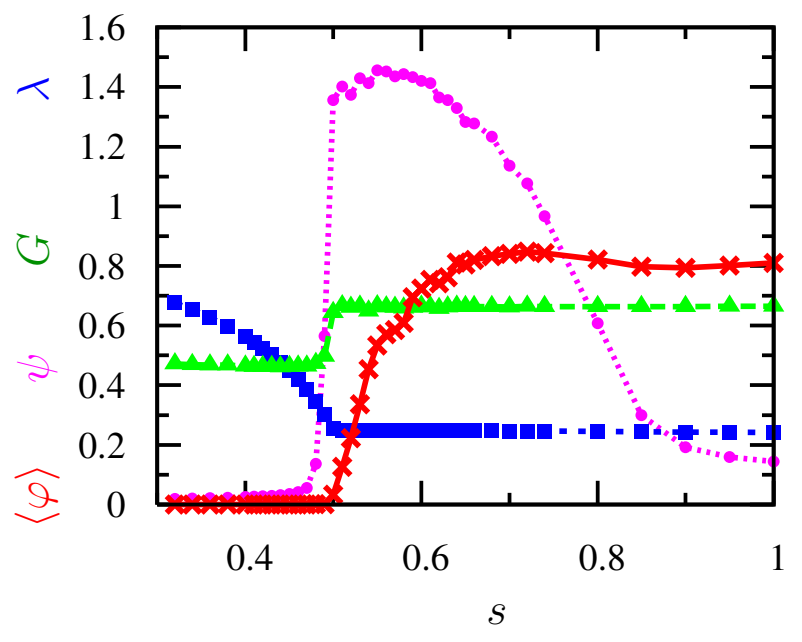

Figure 9: The order parameter ( $\varphi$, x marks), the Binder cumulant ( $G$, triangles), the information exchange between particles ( $\psi$, circles) and the average curvature of trajectories ( $\lambda$, squares) as a function of the strategy variable $s$ in case of the AC-SPP with SVA. Compared to the OVA in case of the SVA the critical strategy value is at lower $s$, but the behavior of the $\varphi, G$ and $\lambda$ functions are very similar. The information exchange between particles has its maximum value near the critical point as well, however, its variability as a function of $s$ is much stronger than for OVA. Parameters as in Figure 1

In the original SPP model [11] (here, following the notation of Huepe and Aldana, Ref [22], called as the Original Vicsek Algorithm (OVA)) the positions of the particles at $t+\Delta t$ are depending on two previous time-steps, $t$ and $t-\Delta t$. In the literature some authors have implemented the model in a slightly different way [20, 28, 29, 30], where the order of the position and the velocity update are changed. Huepe and Aldana [22] refer to this as the Standard Vicsek Algorithm (SVA) and they report that the local density is different in the OVA and the SVA, while in both cases the average number of interacting neighbors is unreasonably high because of the lack of a repulsive effect. By analysing the AC-SPP model with SVA-like updating rule (Figure 9), we find that the behavior of the order parameter, the Binder cumulant and the average curvature are very similar to those in the OVA, but the critical $s$ value is lower. The information exchange rate is, however, rather different and the maximum value of $\psi$ is much higher in case of the SVA. The maximum value of the information exchange compared to the value at $s=1$ in case of the OVA is $\psi_{\max }^{O V A} / \psi_{s=1}^{O V A}=1.4$, while in case of the SVA $\psi_{\max }^{S V A} / \psi_{s=1}^{S V A}=10.1$. At $s=1$, where the AC-SPP is congruous to the original SPP model, the rate of information exchange is similar in the OVA and the SVA.

\section{Conclusions}

In conclusion, we investigated the statistical properties of a three-dimensional self-driven particle system (AC-SPP), designed to be an improved model for the collective motion of living beings and possibly nonliving units (robots). The ordering of particles exhibited a second-order phase transition as a function of the control parameter corresponding to a behavioral strategy in our case.

We found that the information exchange between particles was maximal at the critical point. Due to the important role of information exchange in animal societies, this might indicate that the critical 
point corresponds to an optimal behavioral strategy. In a more general context, this result implies that biological evolution may drive individual traits corresponding to critical values. However, because of the individual based optimality requirement, this possibility needs to be investigated within an evolutionary game theoretical framework.

ACKNOWLEDGMENTS. This work was supported by the EU FP6 Grant "Starflag" and Országos Tudományos Kutatási Alapprogramok (OTKA) 049674.

\section{References}

[1] T. Inagaki, W. Sakamoto, and T. Kuroki, Bull. Jap. Soc. Sci. Fish, 42, 265 (1976).

[2] C. J. Feare, The starlings (Oxford University Press, 1984).

[3] E. Ben-Jacob, I. Cohen, O. Shochet, A. Czirók, and T. Vicsek, Phys. Rev. Lett. 75, 2899 (1995).

[4] E. M. Rauch, M. M. Millonas, and D. R. Chialvo, Phys. Lett. A 207, 185 (1995).

[5] J. A. Shapiro, BioEssays 17, 597 (1995).

[6] J. K. Parrish and L. Edelstein-Keshet, Science 284, 99 (1999).

[7] B. Szabo, G. J. Szollosi, B. Gonci, Z. Juranyi, D. Selmeczi, and T. Vicsek. Phys. Rev. E 74, 061908 (2006).

[8] M. Wu, J. W. Roberts, S. Kim, D. L. Koch, and M.P. DeLisa Applied and Enviromental Microbiology 72, 4987-4994 (2006).

[9] J. K. Parrish and W. M. Hammer, Animal Groups in Three Dimensions (Cambridge University Press, 1997).

[10] I. D. Couzin and J. Krause, Adv. Study Behav. 32, 1-75 (2003).

[11] T. Vicsek, A. Czirók, E. Ben-Jacob, I. Cohen, and O. Shochet, Phys. Rev. Lett. 75, 1226 (1995).

[12] C. W. Reynolds, Computer Graphics 21, 25-33 (1987).

[13] A. Czirók, H. E. Stanley, and T. Vicsek, J. Phys. A 30, 1375 (1997).

[14] Z. Csahók and T. Vicsek, Phys. Rev. E 52, 5297 (1995).

[15] J. Toner and Y. Tu, Phys. Rev. Lett. 75, 4326 (1995).

[16] J. Toner and Y. Tu, Phys. Rev. E 58, 4828 (1998).

[17] J. Toner, Y. Tu, and M. Ulm, Phys. Rev. Lett. 80, 4819 (1998).

[18] A. Czirók, M. Vicsek, and T. Vicsek, Physica A 264, 299-304 (1999).

[19] G. Grégoire, H. Chaté, and Y. Tu, Phys. Rev. E 64, 011902 (2001).

[20] G. Grégoire and H. Chaté, Phys. Rev. Lett. 92, 025702 (2004).

[21] M. Nagy, I. Daruka, and T. Vicsek, Physica A 373, 445-454 (2007). 
[22] C. Huepe and M. Aldana, Physica A. 387, 2809-2822 (2008).

[23] H. Chaté, F. Ginelli, G. Gregoire, and F. Raynaud, Phys. Rev. E 77, 046113 (2008).

[24] M. Ballerini, N. Cabibbo, R. Candelier, A. Cavagna, E. Cisbani, I. Giardina, V. Lecomte, A. Orlandi, G. Parisi, A. Procaccini, M. Viale, and V. Zdravkovic, Proc. Natl. Acad. Sci. USA 105, 1232-1237 (2008).

[25] J. Gautrais, C. Jost, and G. Theraulaz, Ann. Zool. Fennici, in press (2008).

[26] J. Gautrais, C. Jost, M. Soria, A. Campo, S. Motch, R. Fournier, S. Blanco, and G. Theraulaz, J. Math. Biol., in press (2008).

[27] B. Gönci, M. Nagy, and T. Vicsek, EPJ Special Topics 157, 53-59 (2008).

[28] M. Aldana and C. Huepe, J. Stat. Phys. 112, 135-153 (2003).

[29] C. Huepe and M. Aldana, Phys. Rev. Lett. 92, 168701 (2004).

[30] M. Aldana, V. Dossetti, C. Huepe, V.M. Kenkre, and H. Larralde, Phys. Rev. Lett. 98, 095702 (2007).

[31] K. Binder and D. W. Herrmann, Monte Carlo Simulation in Statistical Physics: An Introduction (Springer, Berlin, 1997).

[32] D. Sumpter, J. Buhl, D. Biro, and I. Couzin, Theory Biosci. 127(2), 177-186 (2008). 